\title{
Neuronal avalanches and brain plasticity
}

\author{
L. de Arcangelis*, H.J. Herrmann ${ }^{\dagger}$ and C. Perrone-Capano** \\ ${ }^{*}$ Department of Information Engineering and CNISM, \\ Second University of Naples, 81031 Aversa (CE), Italy \\ ${ }^{\dagger}$ Computational Physics, IfB, ETH-Hönggerberg, \\ Schafmattstr. 6, 8093 Zürich, Switzerland \\ ${ }^{* *}$ Department of Biological Sciences, \\ University of Naples "Federico II", 80134, Naples, Italy
}

\begin{abstract}
Networks of living neurons exhibit an avalanche mode of activity, experimentally found in organotypic cultures. Moreover, experimental studies of morphology indicate that neurons develop a network of small-world-like connections, with the possibility of a very high connectivity degree. Here we discuss a recent model based on self-organized criticality, which consists of an electrical network with threshold firing and activity-dependent synapse strengths. The model is implemented on regular and small world lattices and on a scale-free network, the Apollonian network. The system exhibits an avalanche activity with a power law distribution of sizes and durations. The analysis of the power spectra of the electrical signal reproduces very robustly the power law behaviour with the exponent 0.8 , experimentally measured in electroencephalogram (EEG) spectra. The exponents are found to be quite stable with respect to initial configurations and strength of plastic remodelling, indicating that universality holds for a wide class of neural network models.
\end{abstract}

Keywords: Neuronal activity, Hebbian plasticity, Scale-free networks

PACS: 87.19.La, 05.65.+b, 05.45.Tp, 89.75.-k

\section{INTRODUCTION}

Cortical networks exhibit diverse patters of activity, including oscillations, synchrony and waves. During neuronal activity, each neuron can receive inputs by thousands of other neurons and, when it reaches a threshold, redistributes this integrated activity back to the neuronal network. Recently it has been shown that another mode of activity is neuronal avalanches, with a dynamics similar to self-organized criticality (SOC) $[1,2,3]$, observed in organotypic cultures from coronal slices of rat cortex [4] where neuronal avalanches are stable for many hours [5]. The term SOC usually refers to a mechanism of slow energy accumulation and fast energy redistribution driving the system toward a critical state, where the distribution of avalanche sizes is a power law obtained without fine tuning: no tunable parameter is present in the model.

In order to monitor neural activities, different time series are usually analyzed through power spectra and generally power-law decay is observed. A large number of time series analysis have been performed on medical data that are directly or indirectly related to brain activity. Prominent examples are EEG data which are used by neurologists to discern sleep phases, diagnose epilepsy and other seizure disorders as well as brain damage and disease. Another example of a physiological function which can be monitored by time series analysis is the human gait which is controlled by the brain [6]. For all these time series the power spectrum, i.e. the square of the amplitude of the Fourier trans- 
formation double logarithmically plotted against frequency, generally features a power law at least over one or two orders of magnitude with exponents between 1 and 0.7 . Moreover, experimental results show that neurotransmitter secretion rate exhibits fluctuations with time power law behaviour [7] and power laws are observed in fluctuations of extended excitable systems driven by stochastic fluctuations [8].

Here we discuss a model based on SOC ideas and taking into account synaptic plasticity in a neural network $[9,10]$. Plasticity is one of the most astonishing properties of the brain, occurring mostly during development and learning $[11,12]$, and can be defined as the ability to modify the structural and functional properties of synapses. Modifications in the strength of synapses are thought to underly memory and learning. Among the postulated mechanisms of synaptic plasticity, the activity dependent Hebbian plasticity constitutes the most fully developed and influential model of how information is stored in neural circuits $[13,14]$. A large variety of models for brain activity has been proposed, based for instance on the convolution of oscillators [15] or stochastic waiting times [16]. They are essentially abstract representations on a mesoscopic scale, but none of them is based on the behaviour of a neural network itself. In order to get real insights on the relation between time series and the microscopic, i.e. cellular, interactions inside a neural network, it is necessary to identify the basic ingredients of the brain activity possibly responsible for characteristic scale-free behaviour observed.

\section{NEURONS, SYNAPSES AND HEBBIAN PLASTICITY}

In the early twentieth century the first generation of "modern" neurobiologists had difficulty in interpreting the cellular nature of the nervous system, since this exhibits an extraordinary complexity [17] with respect to other tissues. It is now widely accepted that cells in the nervous system can be divided into two categories: neurons and a variety of supporting cells, as neuroglial cells. Neurons are specialized for electrical signaling over long distances, whereas supporting cells are not capable of electrical signaling, despite having an important role in signal transmission. The human brain is estimated to contain 100 billion neurons and several times as many supporting cells. The interconnection of nerve cells via synapses forms an intricate network, which is the foundation on which sensory processes, perception and behaviour are built.

Each neuron is made of a cell body from which arise one axon and an eventually elaborate arborization of dendrites. These receive inputs from other neurons and represent the most salient morphological feature of nerve cells. Indeed, there exist nerve cells that are connected to just one or few other neurons but also neurons connected up to 100,000 other neurons. The axon is a unique extension from the cell body that may travel a few hundred micrometers or much farther, depending on the type of neuron. The axonal mechanism that carries signals over such distances is called "action potential", a self-regenerating wave of electrical activity that propagates from the cell body to the axon terminal ending with a set of presynaptic buttons. The synaptic terminal is a special secretory apparatus, where typically a presynaptic terminal is adjacent to a postsynaptic specialization of the contacted neuron, with no physical continuity. Indeed the two terminals communicate via secretion of neurotransmitters from the presynaptic terminal that bind to receptors in the postsynaptic specialization. The secretion is triggered by the 
arrival of the action potential to the presynaptic terminal.

The fundamental purpose of neurons is to integrate information from other neurons. The number of inputs is therefore an important determinant of neuronal function. The integrated information is sent out through the axon to synaptic connections with other neurons. The action potential is a signal of the kind "all or none": The membrane resting potential of a neuron is generally $-70 \mathrm{mV}$; if the integrated inputs bring the potential to a threshold value of about $-55 \mathrm{mV}$, the action potential is triggered and, as a consequence, the membrane potential is depolarized. The action potential is a signal of about $100 \mathrm{mV}$ in amplitude and $1-3 m s$ in duration. After sending an action potential, a neuron goes through the so-called "refractory period", during which is unable to answer to any stimulus, regardless its intensity. The refractoriness of the membrane in the wake of the action potential explains why action potentials do not propagate back toward the point of their initiation as they travel along the axon. The arrival of the action potential at the presynaptic buttons gives rise to synaptic transmission usually via chemical synapses: Neurotransmitter is released into the synaptic cleft and can bind to receptor molecules in the postsynaptic membrane, opening postsynaptic ion channels. Depending of the neurotransmitter, the postsynaptic membrane potential can get closer or farther with respect to the threshold potential. Neurotransmitters can then have an excitatory or inhibitory effect.

In 1949 Donald Hebb first proposed that the strength of a synaptic connection depends on the activity of the connection itself [13]. He stated his postulate: "When an axon of cell $\mathrm{A}$ is near enough to excite a cell $\mathrm{B}$ and repeatedly or persistently takes part in firing it, some growth process or metabolic change takes place in one or both cells such that A's efficiency, as one of the cell firing B, is increased". This strengthening has its counterpart in the complement postulate stating that, if a neuron A repeatedly fails to excite neuron $\mathrm{B}$, the strength of the connection is decreased. The pioneering work of Hebb was confirmed only about twenty years later by the recognition of the long term potentiation (LTP) and long term depression (LTD).

Recently a neuronal activity based on avalanches has been observed in organotypic cultures from coronal slices of rat cortex [4] where neuronal avalanches are stable for many hours [5]. More precisely, recording spontaneous local potentials continuously by a multielectrode array, has shown that activity initiated at one electrode might spread to other electrodes not necessarily contiguous, as in a wave-like propagation. Several avalanches per hour of different duration are observed, in which non-synchronous activity is spread over space and time. By analyzing the size and duration of neuronal avalanches, the probability distribution reveals a power law behaviour, suggesting that the cortical network operates in a critical state. The experimental data indicate for the avalanche size distribution a slope -1.5 for optimal experimental conditions. Interestingly, the power law behaviour is destroyed when the excitability of the system is increased, contrary to what expected, since the incidence of large avalanches should decrease the power law exponent. The power law behaviour is therefore the indication of an optimal excitability in the system spontaneous activity. These results have been interpreted relating spontaneous activity in a cortical network to a critical branching process [18], indeed the experimental branching parameter is very close to the critical value equal to one, at which avalanches at all scales exist. Neuronal network simulations with global coupling and static synapses show, for the conservative case, similar values of the 
scaling exponent [19].

In real brain neurons are known to be able to develop an extremely high number of connections with other neurons, that is a single cell body may receive inputs from even a hundred thousand presynaptic neurons. The morphological characterization of a neuronal network grown in vitro has been studied [20] by monitoring the development of neurites in an ensemble of few hundred neurons from the frontal ganglion of adult locusts. After few days the cultured neurons have developed an elaborated network with hundreds of connections, whose morphology and topology has been analyzed by mapping it onto a connected graph. The short path length and the high clustering coefficient measured indicate that the network belongs to the category of small-world networks [21], interpolating between regular and random networks. Moreover, the connectivity degree distribution suggests that the network is "broad scale".

\section{THE MODEL}

In order to formulate a new model to study neuronal activity, we have introduced $[9,10]$ within a SOC approach the three most important ingredients, namely threshold firing, neuron refractory period and activity-dependent synaptic plasticity. We consider a lattice on which each site represents the cell body of a neuron, each bond a synapse. Therefore, on each site we have a potential $v_{i}$ and on each bond a conductance $g_{i j}$ $\left(g_{i j} \neq g_{j i}\right)$. Whenever at time $t$ the value of the potential at a site $i$ is above a certain threshold $v_{i} \geq v_{\max }$, approximately equal to $-55 \mathrm{mV}$ for the real brain, the neuron fires, i.e. generates an "action potential", distributing charges to its connected neighbours in proportion to the current flowing through each bond

$$
v_{j}(t+1)=v_{j}(t)+v_{i}(t) \frac{i_{i j}(t)}{\sum_{k} i_{i k}(t)}
$$

where $v_{j}(t)$ is the potential at time $t$ of site $j$, nearest neighbor of site $i, i_{i j}=g_{i j}\left(v_{i}-v_{j}\right)$ and the sum is extended to all connected sites $k$ of site $i$ that are at a potential $v_{k}<v_{i}$. After firing a neuron is set to a zero resting potential. The conductances are initially all equal to unity whereas the neuron potentials are uniformly distributed random numbers between $v_{\max }-2$ and $v_{\max }-1$. The firing rate of real neurons is limited by the refractory period, i.e. the brief period after the generation of an action potential during which a second action potential is difficult or impossible to elicit. In our model, once a neuron fires, it remains quiescent for one time step and it is therefore unable to accept charge from firing neighbours. This ingredient indeed turns out to be crucial for a controlled functioning of our numerical model.

We have implemented the model on the square lattice of linear size $L$ and on a square lattice with a small fraction of bonds, from 0 to $10 \%$, rewired to long range connections, corresponding to a small world network $[21,20]$, which more realistically reproduces the connections in the real brain. Furthermore, we have also considered the Apollonian network, recently introduced [22]. This network has been found to be both smallworld and scale free and therefore presents the possibility of a very high connectivity degree (scale-free) and connecting bonds of all lengths, features found in real neuronal 
networks. The potential is fixed to zero at the boundary of the different lattices. The external stimulus is imposed either at one input site in the centre of the lattice, or at random in the system, this last choice more closely modeling brain spontaneous activity.

As soon as a site is at or above threshold $v_{\max }$ at a given time $t$, it fires according to Eq. (1), then the conductance of all the bonds, connecting to active neurons and that have carried a current, is increased in the following way

$$
g_{i j}(t+1)=g_{i j}(t)+\delta g_{i j}(t)
$$

where $\delta g_{i j}(t)=k \alpha i_{i j}(t)$, with $\alpha$ being a dimensionless parameter and $k$ a unit constant bearing the dimension of an inverse potential. After applying Eq. (2) the time variable of our simulation is increased by one unit. Eq. (2) describes the mechanism of increase of synaptic strength, tuned by the parameter $\alpha$. This parameter then represents the ensemble of all possible physiological factors influencing synaptic plasticity, many of which are not yet fully understood.

Once an avalanche of firings comes to an end, the conductance of all the bonds with non-zero conductance is reduced by the average conductance increase per bond

$$
\Delta g=\sum_{i j, t} \delta g_{i j}(t) / N_{b}
$$

where $N_{b}$ is the number of bonds with non-zero conductance. The quantity $\Delta g$ depends on $\alpha$ and on the response of the brain to a given stimulus. In this way our electrical network "memorizes" the most used paths of discharge by increasing their conductance, whereas the less used synapses atrophy. Once the conductance of a bond is below an assigned small value $\sigma_{t}$, we remove it, i.e. set it equal to zero, which corresponds to what is known as pruning. These mechanisms correspond to a Hebbian form of activity dependent plasticity, discussed in the previous section.

\section{PRUNING AND NEURONAL AVALANCHES}

The external driving mechanism to the system is imposed by setting the potential of the input site to the value $v_{\max }$, corresponding to one stimulus. This external stimulus is needed to keep functioning the system and therefore mimics the living brain activity. We let the discharge evolve until no further firing occurs, then we apply the next stimulus. We record the total current flowing in the system as function of time during a sequence of successive avalanches. Data show that discharges of all sizes are present in the brain response, as in self-organized criticality where the avalanche size distribution scales as a power law [4].

The strength of the parameter $\alpha$, controlling both the increase and decrease of synaptic strength, determines the plasticity dynamics in the network. In fact, the more the system learns strengthening the used synapses, the more the unused connections will weaken. The number of pruned bonds in the system as function of the number of applied external stimuli indicates that, For large values of $\alpha$ the system strengthens more intensively the synapses carrying current but also very rapidly prunes the less used connections, reaching after a short transient a plateau where it prunes very few bonds. On the contrary, 


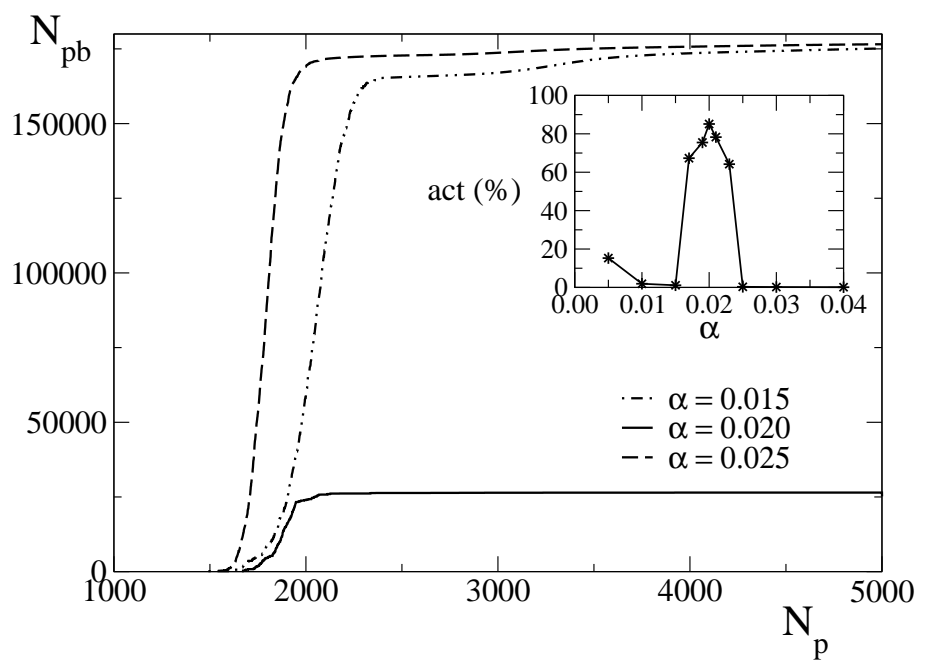

FIGURE 1. The average number of pruned bonds $N_{p b}$ as function of the number of external stimuli $N_{p}$ for the Apollonian network at iteration $N=9$. In the inset, the percentage of the asymptotic number of active bonds as function of $\alpha$. The maximum is found for $\alpha=0.020$.

for small values of $\alpha$ the system takes more time to initiate the pruning process and slowly reaches a plateau (Fig.1). The inset of Fig.1 shows the asymptotic value of the fraction of active bonds, calculated as the total number of bonds in the unpruned network minus the asymptotic number of pruned bonds, as function of $\alpha$. The number of active bonds asymptotically reaches its largest value at a value of $\alpha$, that could be interpreted as an optimal value for the system with respect to plastic adaptation.

Since each avalanche may trigger the activity of a high number of neurons, large currents flow through the system, therefore after $N_{p}$ stimuli the network is no longer the original lattice due to pruning. This complex structure constitutes the first approximation to a trained brain, on which measurements are performed. These consist of a new sequence of stimuli at the input site, by setting the voltage at threshold, during which we measure the number of firing neurons as function of time. This quantity corresponds to the total current flowing in a discharge measured by the electromagnetic signal of the EEG. We have evaluated the size distribution of neural avalanches, that is the total number of neurons involved in the propagation of each stimulus. This distribution exhibit power law behaviour, with an exponent equal to $1.2 \pm 0.1$, quite stable with respect to parameters and lattice type (Fig.2). Conversely, for the input site chosen at random in the system, the scaling exponent changes and becomes $1.5 \pm 0.1$ (Fig.2) in agreement with the experimental value found for spontaneous activity [4]. The scaling exponent for the avalanche duration distribution is also found to be $\tau=2.1 \pm 0.2$, in agreement with the value 2.0 found experimentally by Beggs and Plentz [4].

In order to compare with medical data, we calculate the power spectrum of the resulting time series, i.e. the square of the amplitude of the Fourier transform as function of frequency. The average power spectrum as function of frequency is found (Fig. 3) to exhibit a power law behaviour with the exponent $0.8 \pm 0.1$ over more than three orders of magnitude. This is the same value for the exponent found generically on medical EEG power spectra $[23,24]$. We also show the magnetoelectroencephalography 


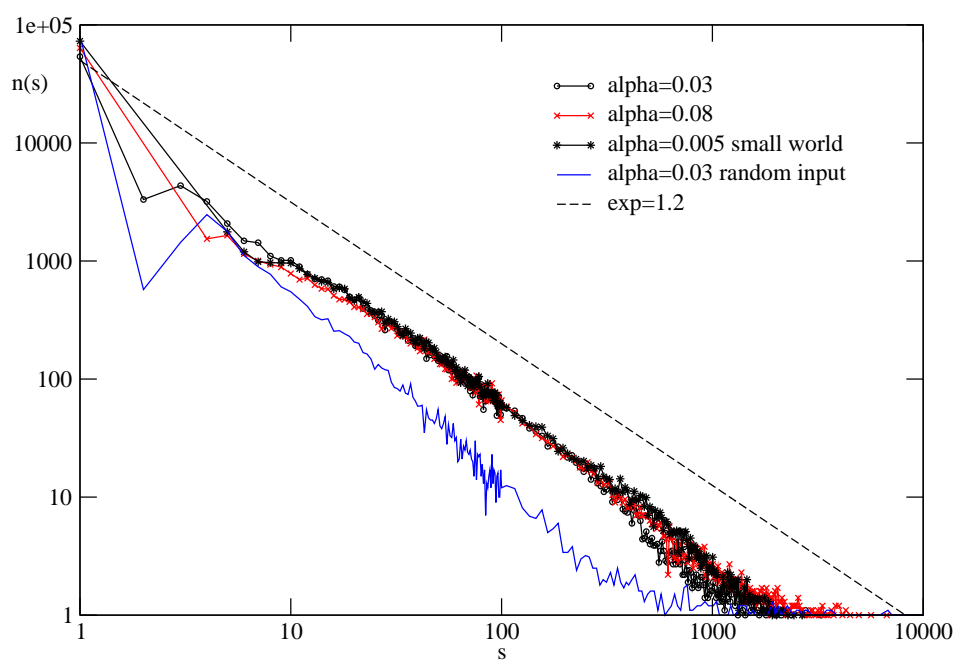

FIGURE 2. (Color online) Log-log plot of the distribution of avalanche size $n(s)(L=1000, \alpha=0.03$ and $0.08, N_{p}=10, v_{\max }=6$ ) for the square lattice (lines) and the small world lattice (*, $L=1000$, $\alpha=0.05, N_{p}=1000, v_{\max }=8$ ) with $1 \%$ rewired bonds. The data are averaged over 10000 stimuli in 10 different configurations and the stimulus is applied at the central site. The dashed line has a slope 1.2. For $\alpha=0.3$ and random input site the slope is 1.5 .

(similar to EEG) obtained from channel 17 in the left hemisphere of a male subject, as measured in ref.[24], having the exponent 0.795 . We have checked that the value of the exponent is stable against changes of the parameters and lattice type. Moreover, the scaling behaviour remains unchanged if the input site is placed at random in the system at each stimulus. Interestingly, when no plasticity mechanism is applied, the spectrum has a behaviour $1 / f$, characteristic of SOC.

\section{CONCLUSIONS}

Extensive simulations have been presented for a novel activity dependent brain model implemented on regular,small world and scale free lattices. The results are compared with experimental data. An interesting result is that an optimal value of of the plasticity strength $\alpha$ exists with respect to the pruning process, that optimize information transmission. Moreover the avalanche size and duration distributions show a power law behaviour, for random input site, in agreement with experimental data.

The stability of the spectrum exponent suggests that an universal scaling characterizes a large class of brain models and physiological signal spectra for brain controlled activities. Medical studies of EEG focus on subtle details of a power spectrum (e.g. shift in peaks) to discern between various pathologies. These detailed structures however live on a background power law spectrum that shows universally an exponent of about 0.8 , as measured for instance in refs. [23] and [24]. A similar exponent was also detected in the spectral analysis of the stride-to-stride fluctuations in the normal human gait which can directly be related to neurological activity [6]. The plasticity of the synapses may be at the origin of the new observed exponent. 


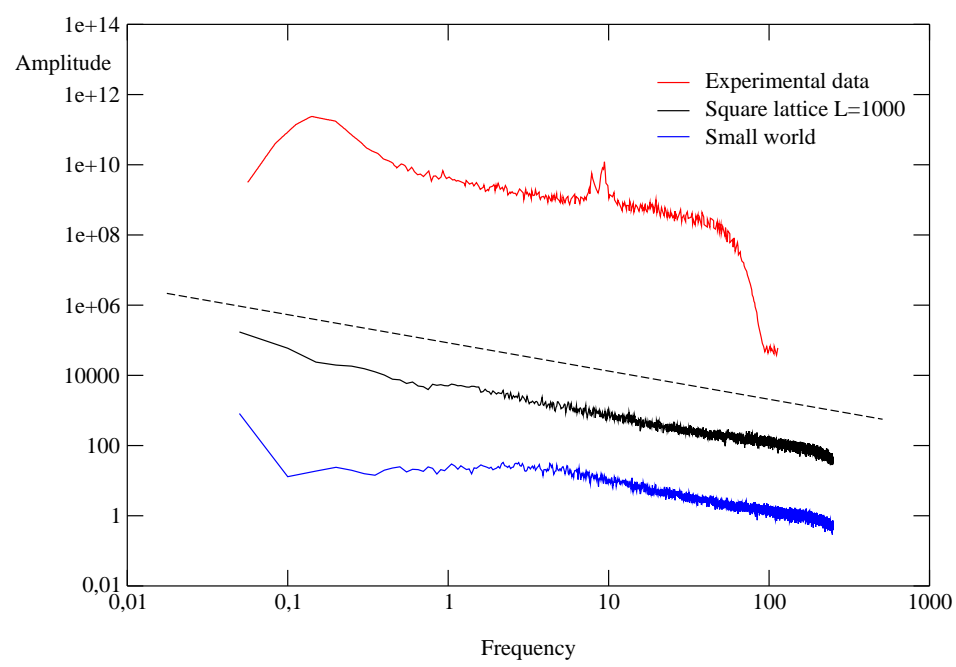

FIGURE 3. (Color online) Power spectra for experimental data and numerical data $(L=1000, \alpha=0.03$, $N_{p}=10, v_{\max }=6$ ) for the square lattice (middle line) and the small world lattice (bottom line, $L=1000$, $\alpha=0.05, N_{p}=1000, v_{\max }=8$ ) with $1 \%$ rewired bonds. The experimental data (top line) are from ref.[25] and frequency is in $\mathrm{Hz}$. The numerical data are averaged over 10000 stimuli in 10 different network configurations. The dashed line has a slope 0.8 .

\section{REFERENCES}

1. P. Bak: How nature works. The science of self-organized criticality, Springer, New York, 1996.

2. S. Maslov, M. Paczuski, P. Bak, Phys. Rev. Lett. 73, 2162-2165 (1994).

3. J. Davidsen, M. Paczuski, Phys. Rev. E 66, 050101(R) (2002).

4. J. M. Beggs, D. Plenz, J. Neurosci. 23, 11167 (2003).

5. J. M. Beggs, D. Plenz, J. Neurosci. 24, 5216 (2004).

6. J. M. Hausdorff et al, Physica A 302, 138 (2001).

7. S.B. Lowen, S.S. Cash, M. Poo , M.C. Teich, J. Neurosci., 17, 5666 (1997).

8. D.R. Chialvo, G.A. Cecchi, M.O. Magnasco, Phys. Rev. E 61, 5654 (2000).

9. L.de Arcangelis, C. Perrone-Capano, H.J. Herrmann, Phys. Rev. Lett. 96, 028107-4 (2006).

10. G.L. Pellegrini, L.de Arcangelis, H.J. Herrmann, C. Perrone-Capano, Phys. Rev. E 76, 016107-9 (2007)

11. T. D. Albraight et al, Neuron, Review supplement to vol. 59 (February 2000).

12. T. K. Hensch, Ann. Rev. Neurosci. 27, 549 (2004).

13. D.O. Hebb, The organization of behaviour, John Wiley, New York, 1949.

14. S.J. Cooper, Neurosci. Biobehav. Rev. 28, 851 (2005).

15. Y. Ashkenazy et al, Physica A 316, 662 (2002).

16. P. Ch. Ivanov et al, Europhys. Letters 43, 363 (1998).

17. D. Purves, G. J. Augustine, D. Fitzpatrick, W. C. Hall, Neuroscience, Sinauer, 2004.

18. S. Zapperi, K.B. Lauritsen, H.E. Stanley, Phys. Rev. Lett. 75, 4071-4074 (1995).

19. C.W. Eurich, J.M. Herrmann, U.A. Ernst, Phys. Rev. E 66, 066137 (2002).

20. O. Shefi, I. Golding, R. Segev, E. Ben-Jacob, A. Ayali, Phys. Rev. E 66, 021905 (2002).

21. D. J. Watts, S. H. Strogatz, Nature 393, 440 (1998).

22. J.S. Andrade, H.J. Herrmann, R.F.S. Andrade, L.R. da Silva, Phys. Rev. Lett. 94, 018702-4 (2005).

23. W. J. Freeman et al, J. Neurosci. Meth. 95, 111 (2000).

24. E. Novikov, A. Novikov, D. Shannahoff-Khalsa, B. Schwartz, J. Wright, Phys. Rev. E 56, R2387 (1997). 